\title{
PerCepCión de la RESPONSABILIDAD SOCIAL UNIVERSITARIA (RSU) POR LOS ALUMNOS DE LA FACULTAD DE ENFERMERÍA Y Nutriología de la Universidad Autónoma de ChiHuahua
}

\author{
Eduardo Morales Loya \\ José Gerardo Reyes López \\ Myrna Isela García Bencomo \\ Universidad Autónoma \\ de Chihuahua
}

RECIBIDO: $1 .^{\circ}$ DE MAYO DE 2017 ACEPTADO: 17 DE AGOSTO DE 2017

\section{RESUMEN}

l objetivo fue evaluar 4 la percepción de la responsabilidad social universitaria (RSU) por los estudiantes de la Facultad de Enfermería y Nutriología (FEN) de la Universidad Autónoma de Chihuahua (UACH). Metodología: El diseño de la investigación fue no experimental transeccional, descriptivo y correlacional; el muestreo, probabilístico. El tamaño de muestra integró 616 alumnos con selección aleatoria. La variable evaluada fue la rsu y se formó con 11 indicadores.
Como instrumento de medición se utilizó el cuestionario "Observando la rsu". Resultados: El 80 \% de los encuestados fueron mujeres, el $62 \%$ registró entre 18 y 20 años de edad, el $81 \%$ no trabaja, y el $65 \%$ se ubicó entre el primero y el cuarto semestre; el $67 \%$ de los estudiantes mencionaron estar de acuerdo o muy de acuerdo con las 66 acciones de 11 indicadores evaluados. Los indicadores institucionales, licenciatura y semestre mostraron relación con 11 y con 10 reactivos, respectivamente. Los indicadores personales (edad, 
sexo, y situación laboral) no tuvieron relación con ningún indicador de la rsu. En promedio, los estudiantes otorgaron una calificación de 8 a la rsu. Solo se encontraron relaciones entre los indicadores sociodemográficos institucionales $\mathrm{y}$ los indicadores de la rsu.

Palabras clave: Responsabilidad social universitaria.

\section{AbSTRACT \\ The objective was to evaluate the percep- tion of university} social responsibility (RSU) by students of the Faculty of Nursing and Nutrition (FEN) of Universidad Autonoma de Chihuahua (UACH). Methodology: The research design was non-experimental transectional descriptive and correlational; sampling was probabilistic. The sample size was 616 students and their selection, random. The variable evaluated was the rsu was composed of 11 indicators. The instrument of measurement was the questionnaire "Observing the rsu". Results: $80 \%$ of the respondents were women; $62 \%$ were between 18 and 20 years of age; $81 \%$ do not work, and $65 \%$ was between the first and fourth semester. $67 \%$ of the students mentioned agreeing or strongly agreeing with the 66 actions of 11 indicators evaluated. Institutional indicators for undergraduate and semester showed relation with 11 and 8 of the 66 reagents respectively. The personal indicators age, sex, and employment status were not related to any rsu indicator. On average, the students awarded a score of 8 to the rsu. Only relationships between institutional sociodemographic indicators and rsu indicators were found.

Keywords: University Social Responsibility.

\section{INTRODUCCIÓN}

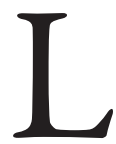

a responsabilidad social (RS) se considera como uno de los pilares de la nueva agenda mundial de la educación superior para potenciar su contribución en la erradicación de la pobreza y el desarrollo sustentable. Según el Pacto Mundial de las Naciones Unidas, el sector educativo puede aumentar el conocimiento y la comprensión de la ciudadanía corporativa. Además, juega un papel importante en la formación de los futuros líderes empresariales y en la educación sobre la importancia de una ciudadanía responsable.

Actualmente, 546 instituciones educativas son parte del Pacto Mundial, y llevan a cabo prácticas de rs y rinden cuentas a través de indicadores previamente establecidos por la onu que son plasmados en un informe público anual, esto permite realizar un benchmarking para mejorar y unificar los criterios a nivel internacional. El problema ante esta realidad es que la universidad tiene un desafío: las demandas del entorno actual plantean la necesidad de buscar, desarrollar y difundir nuevas prácticas en sintonía con estos nuevos escenarios sociales. El enfoque no parte de centralizar la problemática en un aspecto estrictamente técnico, económico o político, ya que la situación abarca, en su dimensión, un problema ético de mayor profundidad y es aquí donde la universidad debe ofrecer sus respuestas para favorecer mecanismos de integración y movilidad social ascendente.

Con base en lo anterior, el objetivo del trabajo fue evaluar la percepción de la rsu por 
los estudiantes de la Facultad de Enfermería y Nutriología (fen) de la uach. Los resultados, de manera general, indican un buen nivel de percepción de la rsu; sin embargo, hay un porcentaje de alumnos que no perciben estas prácticas. Esta investigación se integra en tres secciones básicas: en la primera de ellas se incluye la introducción, el problema y soporte teórico; en la segunda se hace una descripción del proceso metodológico $y$, por último, se presenta la sección de resultados y conclusiones.

\section{JUSTIFICACIÓN}

T a importancia de la rs radica en su papel, cada vez más activo, dentro de las empresas y en las sociedades en las que operan, ya no solamente son generadoras de empleo y riqueza, han pasado a ser un agente fundamental para el desarrollo de las comunidades donde se establecen; ahora son una institución social y motor del cambio y de progreso importante de la sociedad (Flont, Gudiño, Salgado y Sánchez, 2008).

Cuando se ejerce la rs, la atención de una organización se centra en su contribución al desarrollo sustentable, incluyendo la salud y el bienestar de la sociedad. Para ello, el comportamiento de la organización debe ser congruente con principios éticos que sustentan sus acciones y decisiones. Esta consideración reaviva hoy la atención hacia el papel que juegan los principios éticos en la orientación "hacia la sustentabilidad" que pretende ser el compromiso de una organización particular: la universidad pública (Ysunza y Molina, 2010).

Destaca el papel prioritario de la rsu que promueva la gestión social del conocimiento para todos los actores sociales, que tenga como finalidad la construcción de una ciudadanía informada, responsable y participativa, que responda ante la injusticia, la insostenibilidad, la violencia y la corrupción. Repensar la función social de la universidad pública requiere defender los valores propios de la educación pública, en el sentido de que todos los ciudadanos tengan la igualdad de oportunidades para desarrollar sus capacidades, eliminando obstáculos de carácter económico y social, así como los culturales y po- líticos que afectan e impiden ese desarrollo (Gasca y Olvera, 2011).

Las universidades se limitan a ofrecer cifras relacionadas con los principales aspectos de su misión, tales como la cantidad de alumnos matriculados según área de conocimiento y tipo de formación, los artículos científicos y proyectos de investigación desarrollados, o los recursos destinados al desarrollo de diferentes actividades, pero que concretamente no demuestran la magnitud del comportamiento socialmente responsable de cada universidad, a lo sumo son una muestra del proceso de transparencia (Gaete, 2014).

Las universidades están llamadas a ser actores que induzcan los cambios a través de un nuevo perfil de profesionistas caracterizados por la rs. Es insuficiente capacitar en ciencia y tecnología a las nuevas generaciones; se necesita generar agentes que rectifiquen el modelo de desarrollo económico hacia la sustentabilidad, que entiendan y asuman el concepto de rsu (Serna, 2016).

Las universidades son agentes de transformación y consolidación del modelo 
democrático, principalmente ante al reto de construir ciudadanos completos, interesados por las necesidades del entorno físico y social, por la toma de decisiones colectivas, que se conciben a sí mismos como ciudadanos partícipes y no únicamente como votantes con miras a satisfacer sus propios intereses. Con base en esta problemática sobre la importancia de la universidad y del nuevo rol que deben jugar estas instituciones universidades, además de sus funciones sustantivas, se plantearon las siguientes preguntas de investigación, objetivos e hipótesis.

\section{Preguntas de INVESTIGACIÓN}

1) ¿Cómo es la percepción de la rsu por los estudiantes de la fen/uach?

2) ¿Cuál es la relación entre los indicadores sociodemográficos y los indicadores de la rsu?

\section{Objetivos}

1) Evaluar la percepción de la rsu por los estudiantes de la fen de la uach.
2) Identificar la relación entre los indicadores sociodemográficos de los estudiantes de la fen con los indicadores de la rsu.

\section{Hipótesis}

1) La percepción de la rsu por los estudiantes de la fen de la uach es positiva.

2) No existe relación entre los indicadores sociodemográficos de los estudiantes de la fen-uach con los indicadores de la rsu.

\section{Marco teórico}

$n$ el marco del nuevo 4 humanismo pluralista 1 y cosmopolita promovido por la Unesco, a través del Observatorio de Responsabilidad Social Universitaria (ORSALC), se define la rs de las universidades como un compromiso institucional autónomo, pero participativo de personas e instituciones, para orientar el cumplimiento misional hacia la pertinencia social y la gestión ética transparente, de cara a los retos de equidad y a los desafíos ambientales de la sociedad local y global.
La universidad socialmente responsable aspira a la congruencia entre su discurso y sus actos en todos los aspectos de su misión: a) la creación y aplicación de conocimientos para el avance y la cohesión social, fortalecimiento de las identidades culturales, la lucha contra la pobreza, el hambre y las crisis, la generación de pensamiento crítico y de ciudadanía activa; b) la formación de profesionales humanistas comprometidos; c) la gestión de un campus social y ambientalmente ejemplar que construye sinergia entre la autonomía como derecho y condición necesaria de personas y/o instituciones y el cumplimiento de sus propósitos misionales con calidad (modelo de gestión que comprende la rendición pública de cuentas); d) una participación social para contribuir al desarrollo justo y sostenible (Aponte, 2015).

La RSU es una política de mejora continua de la Universidad hacia el cumplimiento efectivo de su misión social mediante cuatro procesos: gestión ética y ambiental de la institución; formación de ciudadanos conscientes y solidarios; producción y difusión 
de conocimientos socialmente pertinentes; participación social en promoción de un desarrollo más equitativo y sostenible. Las estrategias específicas socialmente responsables para lograr esta mejora son: 1) la participación integrada de los grupos de interés internos y externos en el cometido de la Universidad; 2) la articulación de los planes de estudios, la investigación, la extensión y los métodos de enseñanza con la solución de los problemas de la sociedad; 3) el autodiagnóstico regular de la institución con herramientas apropiadas de medición y comunicación para la rendición de cuentas hacia los grupos de interés (Vallaeys, 2008).

El modelo propuesto por el proyecto Universidad construye país es fundamentado en su centro por los principios y valores teniendo tres funciones de la universidad (docencia, investigación y extensión) y sin dejar pasar la actividad de gestión. Los cuatro procesos anteriores deben ser nutridos directamente por los valores y principios que provienen de la rsu. Universidad construye país explica que en el corazón del modelo los principios y

Figura 1. Cuatro tipos de impactos universitarios de la RSU

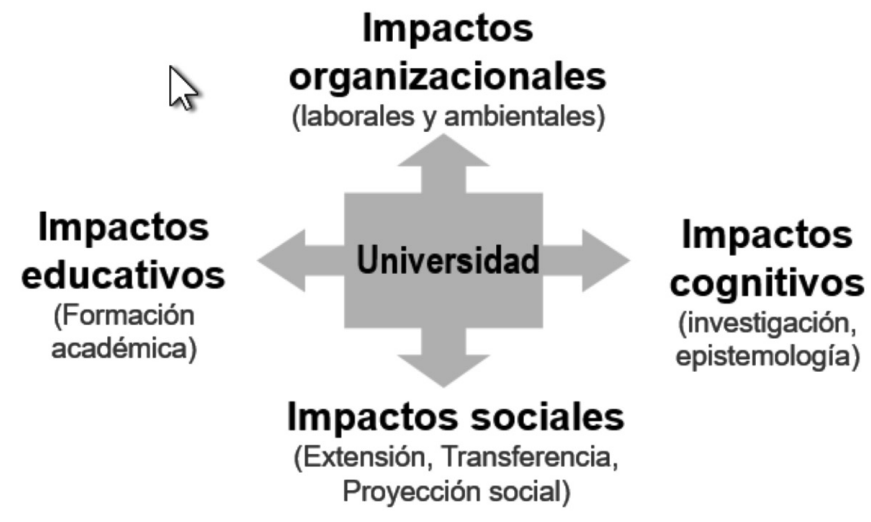

El eje vertical es común a cualquier organización (impactos internos y externos). El eje horizontal es propio de las Universidades, por lo cual la RSU no puede ser equiparada con la RSE, aunque la filosofía que las guía sea la misma.

Fuente: Vallaeys, 2008.

valores generales y los específicos que orientan y constituyen la rsu, se estructuran en una constelación o sistema. Los primeros deben darse en la sociedad para que la universidad pueda desarrollarlos a cabalidad y los segundos podrían considerarse propios de la universidad (Universidad construye país, 2014).

La rsu se define como "la capacidad que tiene la universidad como institución, de difundir y poner en práctica un conjunto de principios y valores generales y específicos por medio de los procesos clave de gestión, docencia, investigación y extensión, respondien- do socialmente ante la comunidad universitaria y el país en que está inserta” (Gaete, 2006). En la figura 1 se aprecian los ejes o impactos que integran la rsu y que pueden ser evaluados por los agentes internos, es este caso, los estudiantes.

\section{Metodología}

lestudio se llevó a cabo
en las instalaciones de
la Fen de la UACH en el periodo de marzo a abril del 2016. El diseño de investigación fue no experimental transeccional descriptivo y correlacional. La población de interés estuvo integrada por los estu- 
diantes de la fen de la uach. El marco muestral se consideró en función de los archivos y la base de datos del departamento académico de la fen. La unidad de análisis estuvo integrada por los estudiantes activos inscritos distribuidos en ocho semestres de dos licenciaturas, Enfermería y Nutrición. La variable evaluada fue la RSU.

Los indicadores medidos fueron: Sociodemográficos, licenciatura, semestre, situación laboral, género y edad; los indicadores de la variable fueron 11: Dignidad de la persona, Libertad, Ciudadanía, participación y democracia, Sociabilidad y solidaridad, Bien común y equidad, Medio ambiente y desarrollo sustentable, Aceptación y aprecio a la diversidad, Compromiso con la verdad, Integridad, Excelencia, Interdependencia e interdisciplina.

El tipo de muestreo fue probabilístico, donde todos los estudiantes de la fen, de los ocho semestres y de las dos licenciaturas, tuvieron la misma oportunidad de ser elegidos para participar en el estudio. El tamaño de muestra se calculó con un $95 \%$ de nivel de confianza y un $5 \%$ de error para cada una de las dos licenciaturas evaluadas. Para la Licenciatura en Nutrición (535) el tamaño de muestra fue de 224 estudiantes. Para Enfermería (834), la muestra fue de 264 alumnos; para una muestra teórica de 488 estudiantes, pero la muestra real fue 686 alumnos. La selección de la muestra fue aleatoria estratificada, considerando a las licenciaturas como estratos. La participación por semestre también fue proporcional.

El instrumento de medición que se utilizó fue el cuestionario "Observando la rsu” del Proyecto Universidad Construye País, adaptado para este estudio, y estuvo integrado por tres secciones: Indicadores sociodemográficos; rsu general; y los 11 indicadores de la rsu. Se integró por 66 preguntas, con cinco opciones de respuesta en escala Likert, desde totalmente de acuerdo hasta totalmente en desacuerdo y "no sé”. Esto permitió conocer el grado de desarrollo de un conjunto de 11 valores y principios que deberían orientar las acciones de gestión, docencia, investigación y extensión de toda universidad y comunidad universitaria que se declare socialmente responsable (Jiménez, De Ferrari, Delpiano, y Ardiles, 2004). El análisis de la información fue descriptivo y correlacional. $\mathrm{Su}$ utilizó la prueba de chi cuadrada para determinar la relación entre los indicadores sociodemográficos y los indicadores de la RSU.

\section{ANÁlisis E INTERPRETACIÓN DE RESULTADOS \\ sta sección está integra- da por los resultados descriptivos y del aná-} lisis de chi cuadrada. El primer cuadro corresponde a los resultados descriptivos sobre los indicadores sociodemográficos de los estudiantes evaluados. El cuadro 2 presenta la apreciación de los estudiantes sobre la rsu. De los cuadros 3 al 15 se incluyen los resultados descriptivos sobre los 11 indicadores de la rsu. Del cuadro 14 al cuadro 20 se presentan los resultados sobre el análisis de chi cuadrada. 
Cuadro 1.- Indicadores sociodemográfico. Estudio de rsu. Facultad de Enfermería y Nutriología de la Universidad Autónoma de Chihuahua, Chihuahua,

México, 2016.

\begin{tabular}{|c|c|c|c|c|c|c|c|c|c|}
\hline \multicolumn{2}{|c|}{ Indicadores } & \multicolumn{8}{|c|}{ Resultados } \\
\hline \multirow{2}{*}{$\begin{array}{l}\text { Indicado- } \\
\text { res institu- } \\
\text { cionales }\end{array}$} & Facultad & \multicolumn{4}{|c|}{$\begin{array}{c}\text { Nutriología } \\
46 \%(314)\end{array}$} & \multicolumn{4}{|c|}{$\begin{array}{l}\text { Enfermería } \\
54 \%(372)\end{array}$} \\
\hline & Semestre & $\begin{array}{c}1 \\
19 \\
\%\end{array}$ & \begin{tabular}{c|}
2 \\
19 \\
$\%$ \\
\end{tabular} & $\begin{array}{c}3 \\
11 \%\end{array}$ & $\begin{array}{c}4 \\
16 \\
\% \\
\end{array}$ & $\begin{array}{c}5 \\
9 \%\end{array}$ & $\begin{array}{c}6 \\
15 \%\end{array}$ & $\begin{array}{c}7 \\
7 \%\end{array}$ & $\begin{array}{c}8 \\
4 \%\end{array}$ \\
\hline \multirow[t]{3}{*}{$\begin{array}{c}\text { Indicado- } \\
\text { res perso- } \\
\text { nales }\end{array}$} & $\begin{array}{c}\text { Rangos de } \\
\text { edad }\end{array}$ & $\begin{array}{c}18 \mathrm{a} \\
62\end{array}$ & & $\begin{array}{c}21 \mathrm{a} \\
23 \\
33 \%\end{array}$ & & & $\begin{array}{c}26 \text { a } 28 \\
1 \%\end{array}$ & $\begin{array}{r}+\mathrm{d} \\
1\end{array}$ & \\
\hline & Sexo & \multicolumn{4}{|c|}{$\begin{array}{c}\text { Hombres } \\
20 \%\end{array}$} & \multicolumn{4}{|c|}{$\begin{array}{c}\text { Mujeres } \\
80 \%\end{array}$} \\
\hline & $\begin{array}{c}\text { Situación } \\
\text { laboral }\end{array}$ & \multicolumn{4}{|c|}{ Sí trabaja $19 \%$} & \multicolumn{4}{|c|}{ No trabaja $81 \%$} \\
\hline
\end{tabular}

Fuente: Elaboración propia.

Cuadro 2. Apreciación de la rsu. Estudio de rsu. Facultad de Enfermería y Nutriología de la Universidad Autónoma de Chihuahua. Chihuahua, México, 2016.

\begin{tabular}{ccccccc}
\hline Escala & 10 & 9 & 8 & 7 & 6 & 5 \\
\hline Apreciación & $4 \%$ & $30 \%$ & $40 \%$ & $15 \%$ & $5 \%$ & $4 \%$ \\
\hline
\end{tabular}

Cuadro 3.- Codificación y categorización de respuestas. Estudio de rsu. Facultad de Enfermería y Nutriología de la Universidad Autónoma de Chihuahua, Chihuahua, México, 2016.

\begin{tabular}{ccccc}
\hline No sé & Nada de acuerdo & Poco de acuerdo & De acuerdo & $\begin{array}{c}\text { Muy } \\
\text { de acuerdo }\end{array}$ \\
\hline 1 & 2 & 3 & 4 & 5 \\
\hline A & B & & C & \\
\hline
\end{tabular}

Fuente: Elaboración propia.

La participación de estudiantes por licenciaturas fue más a menos homogénea con $46 \%$ y $54 \%$ para Nutriología y Enfermería, respectivamen- te. En cuanto a semestres, la distribución fue proporcional para los semestres 1, 2, 3, 4 y con un promedio de participación del $16 \%$ para cada semestre mencionado y el 65 $\%$ se concentró en los primeros cuatro semestres. El $62 \%$ de los jóvenes participantes se ubicó entre los 18 a 20 años de edad. El 81 \% de los estudiantes no trabaja.

Se incluyó la pregunta relacionada con la apreciación de la rsu en la uach. En el cuadro 2 se observa, en función de la escala señalada, que la respuesta no fue contundente y que esta apreciación se concentra en un $85 \%$ entre los valores 7,8 y 9 . El valor promedio se ubicó en el valor 8. Llama la atención que esta respuesta no fue contundente, es decir, no existe una apreciación uniforme, sobre esta práctica.

Para la evaluación de los 11 indicadores, se usó la escala tipo Likert con cinco opciones de respuesta: No sé, Nada de acuerdo, Poco de acuerdo, De acuerdo y Muy de acuerdo. Estas opciones se codificaron con número ascendente del 1 al 5. Posteriormente se generaron tres categorías de respuesta: Categoría A (No sé), categoría B (Nada de acuerdo, Poco de acuerdo) y categoría C (De acuerdo o Muy de acuerdo), como se muestra en el cuadro 3: 
Cuadro 4. Resultados del indicador Dignidad de la persona y sus reactivos. Estudio de rsu. Facultad de Enfermería y Nutriología de la Universidad Autónoma de Chihuahua, Chihuahua, México, 2016.

\begin{tabular}{ccccc}
\hline & Dignidad de la persona & $\%$ & \\
\hline & Reactivo & $\mathrm{A}$ & $\mathrm{B}$ & $\mathrm{C}$ \\
\hline 1 & Da un trato personalizado a cada uno de sus alumnos & 7 & 53 & 40 \\
\hline 2 & $\begin{array}{c}\text { Manifiesta preocupación por el respeto que los profe- } \\
\text { sores dan a sus alumnos }\end{array}$ & 53 & 62 \\
\hline 3 & $\begin{array}{c}\text { Invierte para mantener buenas condiciones de estudio } \\
\text { para sus alumnos }\end{array}$ & 2 & 30 & 68 \\
\hline 4 & Da apoyo personal a los alumnos que están en dificultad & 8 & 33 & 59 \\
\hline 5 & $\begin{array}{c}\text { Exige el respeto a la persona en los trabajos que se en- } \\
\text { carga a los alumnos }\end{array}$ & 7 & 18 & 75 \\
\hline 6 & $\begin{array}{c}\text { Da facilidades para que las alumnas embarazadas o con } \\
\text { hijos pequeños puedan estudiar }\end{array}$ & 17 & 20 & 63 \\
\hline & Promedio & 7 & 31 & 62 \\
\hline
\end{tabular}

Fuente: Elaboración propia.

Cuadro 5. Resultados del indicador Libertad y sus reactivos. Estudio de rsu. Facultad de Enfermería y Nutriología de la Universidad Autónoma de Chihuahua, Chihuahua, México, 2016.

\begin{tabular}{lllll}
\hline & \multicolumn{1}{c}{ Libertad } & \multicolumn{2}{c}{$\%$} \\
\hline & Reactivo & A & B & C \\
\hline 7 & Respeta las decisiones que toman sus alumnos & 8 & 34 & 58 \\
\hline 8 & $\begin{array}{l}\text { Da espacios para que los alumnos expresen libremente } \\
\text { sus propias ideas y creencias }\end{array}$ & 30 & 67 \\
\hline \multirow{2}{*}{$\begin{array}{l}\text { Propicia que los docentes den libertad para que los } \\
\text { alumnos expongan sus pensamientos frente a los pro- } \\
\text { blemas que se ven en clase }\end{array}$} & 1 & 26 & 73 \\
\hline 10 & $\begin{array}{l}\text { Estimula a sus alumnos a asumir libremente compromi- } \\
\text { sos sociales }\end{array}$ & 5 & 23 & 72 \\
\hline 11 & Acoge las ideas o iniciativas de sus alumnos & 6 & 36 & 58 \\
\hline 12 & $\begin{array}{l}\text { Promueve la discusión abierta sobre temas que generan } \\
\text { conflicto en la sociedad }\end{array}$ & 8 & 34 & 58 \\
\hline & Promedio & 5 & 1 & 4 \\
\hline
\end{tabular}

Fuente: Elaboración propia.
Con esta categorización se manejaron los resultados de los 11 indicadores evaluados y así se presentan en los siguientes cuadros:

El cuadro 4 muestra que el $61 \%$ de los alumnos estuvo de acuerdo o muy de acuerdo en que la fen/uach lleva a cabo las acciones referentes al indicador Dignidad de la persona; el $31 \%$ estuvo poco acuerdo o nada de acuerdo, y el $7 \%$ no supo. El reactivo más bajo fue el trato personalizado a los alumnos (40\%), el más alto, exigir respeto hacia los trabajos de los alumnos (75\%).

En este cuadro se muestra que el $64 \%$ de los alumnos estuvo de acuerdo o muy de acuerdo en que la fen/uach lleva a cabo las acciones referentes al indicador Libertad; el $31 \%$ estuvo poco acuerdo o nada de acuerdo; y el $5 \%$ no supo. El reactivo más bajo fue Acoge las idea o iniciativas de los alumnos (57\%). El reactivo más alto fue sobre la libertad de expresar puntos de vista (72\%).

Los resultados de este indicador muestran que el $64 \%$ de los alumnos estuvo de acuerdo o muy de acuerdo en que la fen/uach lleva a cabo las ac- 
Cuadro 6. Resultados del indicador Ciudadanía, participación y democracia, y sus reactivos. Estudio de rsu. Facultad de Enfermería y Nutriología de la

Universidad Autónoma de Chihuahua, Chihuahua, México, 2016.

\begin{tabular}{llccc}
\hline \multicolumn{1}{c}{ Ciudadanía, participación y democracia } & \multicolumn{2}{c}{$\%$} \\
\hline & Reactivo & B & C \\
\hline 13 & $\begin{array}{l}\text { Preocupación por formar ciudadanos que participen } \\
\text { activamente en la sociedad }\end{array}$ & 2 & 78 \\
\hline 14 & $\begin{array}{l}\text { Se interesa en conocer las opiniones e inquietudes de } \\
\text { los alumnos }\end{array}$ & 4 & 50 & 46 \\
\hline 15 & $\begin{array}{l}\text { Recoge los aportes de los alumnos al elaborar sus ma- } \\
\text { llas curriculares }\end{array}$ & 24 & 34 & 42 \\
\hline 16 & $\begin{array}{l}\text { Incentiva que los alumnos participen en los orga- } \\
\text { nismos que los representan, tal como los centros de } \\
\text { alumnos }\end{array}$ & 9 & 27 & 64 \\
\hline 17 & $\begin{array}{l}\text { Le interesa formar a los alumnos en el respeto de los } \\
\text { derechos humanos }\end{array}$ & 2 & 14 & 84 \\
\hline 18 & $\begin{array}{l}\text { Contribuye a formar opinión sobre temas públicos } \\
\text { relevantes para la comunidad }\end{array}$ & 7 & 24 & 69 \\
\hline & \begin{tabular}{l} 
Promedio \\
\hline
\end{tabular} & 8 & 28 & 64 \\
\hline
\end{tabular}

Fuente: Elaboración propia.

Cuadro 7. Resultados del indicador Sociabilidad y solidaridad, y sus reactivos. Estudio de rsu. Facultad de Enfermería y Nutriología de la Universidad Autónoma de Chihuahua, Chihuahua, México, 2016.

\begin{tabular}{llccc}
\hline & \multicolumn{1}{c}{ Sociabilidad y solidaridad } & \multicolumn{2}{c}{$\%$} \\
\hline & Reactivo & A & B & C \\
\hline 19 & $\begin{array}{l}\text { Incentiva a los alumnos a prestar servicios a personas, } \\
\text { grupos o comunidades de escasos recursos }\end{array}$ & 6 & 22 & 72 \\
\hline 20 & Estimula la solidaridad entre los alumnos & 2 & 20 & 78 \\
\hline 21 & $\begin{array}{l}\text { Promueve un trato respetuoso a todas las personas, sin } \\
\text { distinción }\end{array}$ & 1 & 14 & 85 \\
\hline 22 & $\begin{array}{l}\text { Desarrolla actividades de integración entre alumnos, } \\
\text { profesores y funcionarios }\end{array}$ & 4 & 28 & 68 \\
\hline 23 & $\begin{array}{l}\text { Orienta la formación de los alumnos para que vean en } \\
\text { su profesión una instancia de servicio a los demás }\end{array}$ & 4 & 17 & 79 \\
\hline 24 & $\begin{array}{l}\text { Da importancia al desarrollo de las habilidades sociales } \\
\text { de sus alumnos (capacidad de escucha, comunicación } \\
\text { efectiva, empatía, etcétera) }\end{array}$ & 4 & 25 & 71 \\
\hline & Promedio & 4 & 21 & 75 \\
\hline
\end{tabular}

Fuente: Elaboración propia. ciones referentes al indicador Ciudadanía, participación, y democracia; el $28 \%$ estuvo poco acuerdo o nada de acuerdo; y el $8 \%$ no supo. El reactivo más bajo fue recoger aportes de los alumnos al elaborar mallas curriculares (41\%). El reactivo más alto fue interesa formar a los alumnos en el respeto de los derechos humanos (84\%).

Se observa que el $75 \%$ de los alumnos estuvo de acuerdo o muy de acuerdo en que la uach/fen lleva a cabo las acciones referentes al indicador Sociabilidad y solidaridad; el $21 \%$ estuvo poco acuerdo o nada de acuerdo; y el $4 \%$ no supo. El reactivo más bajo fue Desarrollar actividades de integración entre alumnos, profesores y funcionarios (67\%). El reactivo más alto fue en el indicador Promueve un trato respetuoso a todas las personas, sin distinción ( $85 \%$ ).

Estos resultados fueron de los más bajos. Se puede apreciar que el $54 \%$ de los alumnos estuvo de acuerdo o muy de acuerdo en que la uach/ fen lleva a cabo las acciones de Bien común y equidad; el $30 \%$ estuvo poco de acuerdo o nada de acuerdo; y el $15 \%$ 
Cuadro 8. Resultados del indicador Bien común y equidad, y sus reactivos. Estudio de rsu. Facultad de Enfermería y Nutriología de la Universidad Autónoma de Chihuahua. Chihuahua, Chihuahua, México, 2016

\begin{tabular}{lllll}
\hline & \multicolumn{1}{c}{ Bien común y equidad } & \multicolumn{2}{c}{$\%$} \\
\hline & Reactivo & A & B & C \\
\hline 25 & Asigna con equidad sus recursos entre las unidades & 18 & 36 & 46 \\
\hline 26 & $\begin{array}{l}\text { Da importancia a la equidad y la justicia social en } \\
\text { formación de sus alumnos }\end{array}$ & 6 & 25 & 69 \\
\hline 27 & $\begin{array}{l}\text { Da facilidades a alumnos de escasos recursos para que } \\
\text { ingresen a ella }\end{array}$ & 9 & 31 & 60 \\
\hline 28 & $\begin{array}{l}\text { Tiene un sistema para ayudar a resolver los problemas } \\
\text { económicos de sus alumnos de escasos recursos }\end{array}$ & 16 & 31 & 53 \\
\hline 29 & $\begin{array}{l}\text { Da apoyos especiales a los alumnos que tienen proble- } \\
\text { mas de rendimiento }\end{array}$ & 25 & 33 & 42 \\
\hline 30 & $\begin{array}{l}\text { Da apoyo especiales a los alumnos que tienen proble- } \\
\text { mas emocionales o psicológicos }\end{array}$ & 19 & 27 & 54 \\
\hline & Promedio & 15 & 30 & 55 \\
\hline
\end{tabular}

Fuente: Elaboración propia.

Cuadro 9. Resultados del indicador Medio ambiente y desarrollo sustentable, y sus reactivos. Estudio de rsu. Facultad de Enfermería y Nutriología de la

Universidad Autónoma de Chihuahua, Chihuahua, México, 2016.

\begin{tabular}{|c|c|c|c|c|}
\hline & \multirow{2}{*}{$\begin{array}{l}\text { Medio ambiente y desarrollo sustentable } \\
\text { Reactivo }\end{array}$} & \multicolumn{3}{|c|}{$\%$} \\
\hline & & A & B & $\mathrm{C}$ \\
\hline 31 & $\begin{array}{l}\text { Capacita a sus miembros en el autocuidado, informan- } \\
\text { do acerca de cómo las personas pueden prevenir ciertas } \\
\text { enfermedades }\end{array}$ & 6 & 23 & 71 \\
\hline 32 & $\begin{array}{l}\text { Tiene una preocupación especial por la formación de } \\
\text { sus alumnos en el cuidado del medio ambiente }\end{array}$ & 3 & 31 & 66 \\
\hline 33 & $\begin{array}{l}\text { Incentiva a sus miembros a economizar el agua, la ener- } \\
\text { gía, etcétera. }\end{array}$ & 6 & 50 & 44 \\
\hline 34 & $\begin{array}{l}\text { Establece normas que limitan el consumo de cigarrillos } \\
\text { en espacios públicos cerrados }\end{array}$ & 1 & 15 & 84 \\
\hline 35 & $\begin{array}{l}\text { Dispone de depósitos para separar la basura según tipo } \\
\text { de material (vidrio, papel, otros) }\end{array}$ & 1 & 7 & 92 \\
\hline 36 & $\begin{array}{l}\text { Crea conciencia en la comunidad Universitaria de los } \\
\text { problemas ambientales que enfrenta nuestra sociedad }\end{array}$ & 3 & 31 & 66 \\
\hline & Promedio & 3 & 26 & 71 \\
\hline
\end{tabular}

Fuente: Elaboración propia. no supo. El reactivo más bajo fue $\mathrm{Da}$ apoyos especiales a los alumnos que tienen problemas de rendimiento (42\%). El reactivo más alto fue $\mathrm{Da}$ importancia a la equidad y la justicia social en formación de sus alumnos (69\%).

Este indicador indica que el $70 \%$ estuvo de acuerdo o muy de acuerdo en que la uach/fen lleva a cabo acciones de Medio ambiente y desarrollo sustentable; el $26 \%$ estuvo poco acuerdo o nada de acuerdo, y el $3 \%$ no supo. El reactivo más bajo fue Incentiva a sus miembros a economizar el agua, la energía, etcétera (43\%). El reactivo más alto fue dispone de depósitos para separar la basura según tipo de material (92\%).

El cuadro 10 muestra que el $66 \%$ de los alumnos estuvo de acuerdo o muy de acuerdo en que la uach/fen lleva a cabo las acciones de Aceptación y aprecio a la diversidad; el 23 $\%$ estuvo poco acuerdo o nada de acuerdo y el $11 \%$ no supo. El reactivo más bajo fue Realiza foros y paneles para que sus miembros conozcan las distintas posturas que hay frente a problemas de interés nacional (41\%). El reactivo más alto 
Cuadro 10. Resultados del indicador Aceptación y aprecio a la diversidad, y sus reactivos. Estudio de rsu. Facultad de Enfermería y Nutriología de la Universidad Autónoma de Chihuahua, México, 2016.

\begin{tabular}{llccc}
\hline \multicolumn{1}{c}{ Aceptación y aprecio a la diversidad } & \multicolumn{2}{c}{$\%$} \\
\hline & Reactivo & A & B & C \\
\hline 37 & $\begin{array}{l}\text { Actúa con respeto por la diversidad humana que hay } \\
\text { en ella (razas, nacionalidades, culturas, etcétera) }\end{array}$ & 3 & 10 & 87 \\
\hline 38 & $\begin{array}{l}\text { Facilita la posibilidad de expresión de las diversas } \\
\text { tendencias religiosas de sus miembros }\end{array}$ & 12 & 22 & 66 \\
\hline 39 & $\begin{array}{l}\text { Se preocupa de que las diversas tendencias políticas } \\
\text { de sus miembros sean respetadas }\end{array}$ & 16 & 28 & 56 \\
\hline 40 & $\begin{array}{l}\text { Realiza foros y paneles para que sus miembros conoz- } \\
\text { can las distintas posturas que hay frente a problemas } \\
\text { de interés nacional }\end{array}$ & 19 & 39 & 42 \\
\hline 41 & $\begin{array}{l}\text { Da facilidad de acceso a ella a grupos especiales (dis- } \\
\text { capacitados, indígenas. etcétera) }\end{array}$ & 12 & 17 & 71 \\
\hline 42 & $\begin{array}{l}\text { Posee infraestructura especialmente adecuada para } \\
\text { discapacitados }\end{array}$ & 2 & 21 & 77 \\
\hline & Promedio & 11 & 23 & 66 \\
\hline
\end{tabular}

Fuente: Elaboración propia.

Cuadro 11. Resultados del indicador Compromiso con la verdad, y sus reactivos. Estudio de rsu. Facultad de Enfermería y Nutriología de la Universidad Autónoma de Chihuahua, Chihuahua, México, 2016.

\begin{tabular}{llccc}
\hline \multicolumn{1}{c}{ Compromiso con la verdad } & \multicolumn{2}{c}{$\%$} \\
\hline Reactivo & A & B & C \\
\hline 43 & $\begin{array}{l}\text { Realiza su enseñanza con un fuerte apego a la verdad } \\
\text { sin acomodarla ni ocultarla para satisfacer intereses } \\
\text { particulares }\end{array}$ & 29 & 62 \\
\hline 44 & $\begin{array}{l}\text { Desarrolla el espíritu crítico de sus alumnos a través } \\
\text { de sus actividades de aprendizaje }\end{array}$ & 4 & 21 & 75 \\
\hline 45 & $\begin{array}{l}\text { Realiza una comunicación interna honesta y transpa- } \\
\text { rente }\end{array}$ & 7 & 35 & 58 \\
\hline 46 & $\begin{array}{l}\text { Realiza un marketing honesto y transparente hacia la } \\
\text { comunidad }\end{array}$ & 13 & 35 & 52 \\
\hline 47 & $\begin{array}{l}\text { Da reconocimiento al compromiso con la verdad de } \\
\text { sus miembros }\end{array}$ & 12 & 29 & 59 \\
\hline 48 & Estimula las ideas creativas de sus alumnos & 5 & 27 & 68 \\
\hline & Promedio & 8 & 29 & 63 \\
\hline
\end{tabular}

Fuente: Elaboración propia. fue Actúa con respeto por la diversidad humana que hay en ella (87\%).

El indicador Verdad, señala que el $63 \%$ de los alumnos estuvo de acuerdo o muy de acuerdo en que la uach/fen lleva a cabo las acciones de Compromiso con la verdad; el 29 $\%$ estuvo poco acuerdo o nada de acuerdo y el $8 \%$ no supo. El reactivo más bajo fue el número 46 con $52 \%$ y el reactivo más alto fue el 44 con $74 \%$ de aprobación.

El cuadro 12 se observa que el $70 \%$ de los alumnos estuvo de acuerdo o muy de acuerdo en que la uach/fen lleva a cabo las acciones de Integridad; el $25 \%$ estuvo poco acuerdo o nada de acuerdo, y el $5 \%$ no supo. El reactivo más bajo fue Sus autoridades muestran coherencia entre sus principios y sus acciones $(63 \%)$. El reactivo más alto fue Promueve que los alumnos respeten la propiedad intelectual (79\%).

El $76 \%$ de los alumnos estuvo de acuerdo o muy de acuerdo en que la $\mathrm{UACH}$ FEN lleva a cabo las acciones de Excelencia; el 18 \% estuvo poco acuerdo o nada de acuerdo; y el $6 \%$ no supo. El reactivo más bajo fue Promueve la 
Cuadro 12. Resultados del indicador Integridad y sus reactivos. Estudio de rsu. Facultad de Enfermería y Nutriología de la Universidad Autónoma de Chihuahua. Chihuahua, México, 2016

\begin{tabular}{llccc}
\hline \multicolumn{1}{c}{ Integridad } & \multicolumn{2}{c}{$\%$} \\
\hline Reactivo & A & B & C \\
\hline 49 & $\begin{array}{l}\text { Cuenta con criterios éticos escritos y conocidos } \\
\text { por la universidad para orientar las conductas de } \\
\text { sus miembros }\end{array}$ & 9 & 17 & 74 \\
\hline 50 & $\begin{array}{l}\text { Sus autoridades muestran coherencia entre sus } \\
\text { principios y sus acciones }\end{array}$ & 4 & 32 & 64 \\
\hline 51 & $\begin{array}{l}\text { Los docentes son estrictos frente a conductas de } \\
\text { deshonestidad académica de sus alumnos }\end{array}$ & 5 & 26 & 69 \\
\hline 52 & $\begin{array}{l}\text { La conducta de los docentes es coherente con los } \\
\text { criterios éticos que tiene la universidad }\end{array}$ & 4 & 30 & 66 \\
\hline 53 & $\begin{array}{l}\text { Cumple los compromisos contraídos con sus } \\
\text { alumnos }\end{array}$ & 6 & 29 & 65 \\
\hline 54 & $\begin{array}{l}\text { Promueve que los alumnos respeten la propiedad } \\
\text { intelectual }\end{array}$ & 4 & 17 & 79 \\
\hline & Promedio & 5 & 25 & 70 \\
\hline
\end{tabular}

Fuente: Elaboración propia.

Cuadro 13. Resultados del indicador Excelencia, y sus reactivos. Estudio de rsu. Facultad de Enfermería y Nutriología de la Universidad Autónoma de Chihuahua, Chihuahua, México, 2016.

\begin{tabular}{llccc}
\hline \multicolumn{1}{c}{ Excelencia } & \multicolumn{2}{c}{$\%$} \\
\hline & Reactivo & A & B & C \\
\hline 55 & $\begin{array}{l}\text { Imparte una formación profesional e intelectual de } \\
\text { alto nivel a sus alumnos }\end{array}$ & 24 & 74 \\
\hline 56 & $\begin{array}{l}\text { Tiene una política de docencia que promueve el "de- } \\
\text { sarrollo integral” de los alumnos }\end{array}$ & 6 & 19 & 75 \\
\hline 57 & $\begin{array}{l}\text { Usa sistemas para que los alumnos evalúen el desem- } \\
\text { peño docente }\end{array}$ & 2 & 9 & 89 \\
\hline 58 & $\begin{array}{l}\text { Promueve la innovación y actualización de los currí- } \\
\text { culos }\end{array}$ & 16 & 19 & 65 \\
\hline 59 & $\begin{array}{l}\text { Promueve la innovación de los métodos de enseñanza } \\
60\end{array}$ & $\begin{array}{l}\text { Presta servicios de apoyo a la docencia (computación, } \\
\text { bibliotecas, tutorías, etcétera) que son de alta calidad }\end{array}$ & 22 & 70 \\
\hline & Promedio & 6 & 18 & 76 \\
\hline
\end{tabular}

Fuente: Elaboración propia. innovación y actualización de los currículos (65\%). El reactivo más alto fue Presta servicios de apoyo a la docencia (computación, bibliotecas, tutorías, etcétera) que son de alta cali$\operatorname{dad}(85 \%)$.

Con este último indicador se detectó que el $72 \%$ de los alumnos estuvo de acuerdo o muy de acuerdo en que la uach/fen lleva a cabo las acciones de Interdependencia e interdisciplina; el $20 \%$ estuvo poco acuerdo o nada de acuerdo; y el $8 \%$ no supo. El reactivo más bajo: Tiene mallas curriculares que exigen a los alumnos tomar cursos de otras disciplinas (59\%). El reactivo más alto fue Incentiva el trabajo en equipo de sus alumnos (84\%).

En este cuadro se aprecia que el $67 \%$ de los estudiantes están de acuerdo o muy de acuerdo en las políticas y/o prácticas de RSU, lo cual se puede considerar como bueno. Por otro lado, llama la atención que el $26 \%$ de ellos no perciben lo mismo, es decir, no están nada de acuerdo o poco de acuerdo con estas actividades, sumados al $7 \%$ que no sabe. Se puede asumir que de cada 10 estudiantes, 
Cuadro 14. Resultados del indicador Interdependencia e interdisciplina, y sus reactivos. Estudio de rsu. Facultad de Enfermería y Nutriología de la Universidad Autónoma de Chihuahua, Chihuahua, México, 2016.

\begin{tabular}{llccc}
\hline \multicolumn{1}{c}{ Interdependencia e interdisciplina } & $\%$ & \\
\hline & Reactivo & $\mathrm{A}$ & $\mathrm{B}$ & $\mathrm{C}$ \\
\hline 61 & $\begin{array}{l}\text { Promueve que los cursos tengan un enfoque interdis- } \\
\text { ciplinario }\end{array}$ & 16 & 75 \\
\hline 62 & $\begin{array}{l}\text { Tiene mallas curriculares que exigen a los alumnos } \\
\text { tomar cursos de otras disciplinas }\end{array}$ & 15 & 26 & 59 \\
\hline 63 & Incentiva el trabajo en equipo de sus alumnos & 3 & 13 & 84 \\
\hline 64 & $\begin{array}{l}\text { Busca solucionar los problemas que se presentan con } \\
\text { la participación de las personas o estamentos directa- } \\
\text { mente afectados }\end{array}$ & 8 & 22 & 70 \\
\hline 65 & $\begin{array}{l}\text { Propicia que las actividades académicas traten los } \\
\text { desafíos o problemas que enfrenta nuestra sociedad }\end{array}$ & 6 & 22 & 72 \\
\hline 66 & $\begin{array}{l}\text { Promueve el vínculo de las actividades Universitarias } \\
\text { con la comunidad local }\end{array}$ & 6 & 22 & 72 \\
\hline & Promedio & 8 & 20 & 72 \\
\hline
\end{tabular}

Fuente: Elaboración propia.

Cuadro 15. Resultados rsu por Indicador y Generales. Estudio de rsu. Facultad de Enfermería y Nutriología de la Universidad Autónoma de Chihuahua, Chihuahua, México, 2016.

\begin{tabular}{|c|c|c|c|c|c|}
\hline \multirow[b]{2}{*}{ Indicador RSU } & \multicolumn{5}{|c|}{ Opciones de respuesta (\%) } \\
\hline & No sé & $\begin{array}{l}\text { Nada de } \\
\text { acuerdo }\end{array}$ & $\begin{array}{l}\text { Poco de } \\
\text { acuerdo }\end{array}$ & $\begin{array}{c}\text { De } \\
\text { acuerdo }\end{array}$ & $\begin{array}{l}\text { Muy de } \\
\text { acuerdo }\end{array}$ \\
\hline 1. Dignidad de la persona & 7 & & & & \\
\hline 2. Libertad & 5 & & & & \\
\hline $\begin{array}{l}\text { 3. Ciudadanía, participación y } \\
\text { democracia }\end{array}$ & 8 & & & & 4 \\
\hline 4. Sociabilidad y solidaridad & 4 & & & & 5 \\
\hline 5. Bien común y equidad & 15 & & & & 5 \\
\hline $\begin{array}{l}\text { 6. Medio ambiente y desarro- } \\
\text { llo sustentable }\end{array}$ & 3 & & & & 1 \\
\hline $\begin{array}{l}\text { 7. Aceptación y aprecio a la } \\
\text { diversidad }\end{array}$ & 11 & & & & 6 \\
\hline 8. Compromiso con la verdad & 8 & & & & 3 \\
\hline 9. Integridad & 5 & & & & 0 \\
\hline 10. Excelencia & 6 & & & & 6 \\
\hline $\begin{array}{l}\text { 11. Interdependencia e inter- } \\
\text { disciplina }\end{array}$ & 8 & & & & 2 \\
\hline Promedio & 7 & & & & 7 \\
\hline
\end{tabular}

Fuente: Elaboración propia. aproximadamente 7 de ellos coinciden con las políticas institucionales y 3 de ellos, no lo hacen.

\section{Resultados DE LA PRUEBA DE CHI CUADRADA}

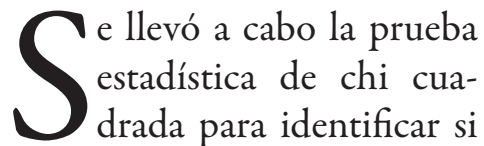
los indicadores sociodemográficos (institucionales y personales, variable independiente) y los indicadores de la RsU (variable dependiente) tienen relación.

La hipótesis para la prueba de chi cuadrada se definió de la siguiente manera:

$\mathrm{H}_{0:}$ Los indicadores sociolaborales y los indicadores de la rsu son independientes; es decir, no hay ninguna relación entre ellas.

$\mathrm{H}_{1:}$ Los indicadores sociolaborales y los indicadores de la rsu son dependientes; es decir, hay relación entre ellas.

La regla de decisión es: se rechaza la $H_{o}$ : si >

Los resultados aceptados son los que tienen un valor alfa inferior a 0.01 (nivel de significancia del $99 \%$ ) y siempre que el número de celdas con 
Cuadro 16. Prueba chi cuadrada entre los indicadores sociodemográfico (licenciatura) y los indicadores de la RSU. Estudio de rsu. Facultad de Enfermería y Nutriología de la Universidad Autónoma de Chihuahua, Chihuahua, México, 2016.

\begin{tabular}{|c|c|c|c|c|c|}
\hline \multicolumn{6}{|c|}{ Variable independiente indicador institucional: Licenciatura } \\
\hline & \multicolumn{2}{|l|}{ Variables dependientes } & \multirow{2}{*}{$\mathrm{X}^{2}$} & \multirow{2}{*}{ gl } & \multirow{2}{*}{$\mathrm{X}^{2} \mathrm{u}$} \\
\hline & Indicador RSU & Reactivo & & & \\
\hline 1 & 1. Dignidad de la Persona & $\begin{array}{l}\text { 6. Da facilidades para que las alumnas embarazadas o con hijos } \\
\text { pequeños puedan estudiar }\end{array}$ & 21282 & 4 & 0,000 \\
\hline 2 & 2. Libertad & $\begin{array}{l}\text { 19. Propicia que los docentes den libertad para que los alumnos } \\
\text { expongan sus pensamientos frente a los problemas que se ven } \\
\text { en clase }\end{array}$ & 13439 & 4 & 0,009 \\
\hline 3 & 2. Libertad & $\begin{array}{l}\text { 12. Promueve la discusión abierta sobre temas que generan } \\
\text { conflicto en la sociedad }\end{array}$ & 13079 & 4 & 0,001 \\
\hline 4 & $\begin{array}{l}\text { 3. Ciudadanía, participa- } \\
\text { ción y democracia }\end{array}$ & $\begin{array}{l}\text { 15. Recoge los aportes de los alumnos al elaborar sus mallas } \\
\text { curriculares }\end{array}$ & 19369 & 4 & 0,001 \\
\hline 5 & 5. Bien común y equidad & $\begin{array}{l}\text { 30. Da apoyo especiales a los alumnos que tienen problemas } \\
\text { emocionales o psicológicos }\end{array}$ & 23269 & 4 & 0,000 \\
\hline 6 & $\begin{array}{l}\text { 6. Medio Ambiente y } \\
\text { Desarrollo Sustentable }\end{array}$ & $\begin{array}{l}\text { 31. Capacita a sus miembros en el autocuidado, informando } \\
\text { acerca de cómo las personas pueden prevenir ciertas enferme- } \\
\text { dades }\end{array}$ & 34114 & 4 & 0,000 \\
\hline 7 & $\begin{array}{l}\text { 7. Aceptación y Aprecio a } \\
\text { la Diversidad }\end{array}$ & $\begin{array}{l}\text { 41. Da facilidad de acceso a ella a grupos especiales (discapaci- } \\
\text { tados, indígenas. etc.) }\end{array}$ & 13397 & 4 & 0,009 \\
\hline 8 & 9. Integridad & $\begin{array}{l}\text { 52. La conducta de los docentes es coherente con los criterios } \\
\text { éticos que tiene la universidad }\end{array}$ & 15014 & 4 & 0,005 \\
\hline 9 & $\begin{array}{l}\text { 11. Interdependencia e } \\
\text { Interdisciplina }\end{array}$ & 68. Incentiva el trabajo en equipo de sus alumnos & 13596 & 4 & 0,007 \\
\hline 10 & $\begin{array}{l}\text { 11. Interdependencia e } \\
\text { Interdisciplina }\end{array}$ & $\begin{array}{l}\text { 64. Busca solucionar los problemas que se presentan con la par- } \\
\text { ticipación de las personas o estamentos directamente afectados }\end{array}$ & 15191 & 4 & 0,004 \\
\hline 11 & $\begin{array}{l}\text { 11. Interdependencia e } \\
\text { Interdisciplina }\end{array}$ & $\begin{array}{l}\text { 65. Propicia que las actividades académicas traten los desafíos o } \\
\text { problemas que enfrenta nuestra sociedad: }\end{array}$ & 14772 & 4 & 0,005 \\
\hline
\end{tabular}

Fuente: Elaboración propia.

frecuencia esperada inferior a 5 no supere el $20 \%$ del total de celdas de la tabla de contingencia.

Se realizó el análisis correspondiente y de identificó que solo los indicadores institucio- nales (licenciatura y semestre) tuvieron relación con algunos de los indicadores de la RSU. Por otro lado, los indicadores individuales como la edad, sexo y la situación laboral, nin- guno de ellos mostró relación con los reactivos de la RSU.

En este cuadro se aprecian las 11 relaciones del indicador Licenciatura con los nueve indicadores de la rsu y específicamente con cada uno de 
Cuadro 17.- Prueba chi cuadrada entre los indicadores sociodemográfico (semestre) y los indicadores de la rsu. Estudio de rsu. Facultad de Enfermería y Nutriología de la Universidad Autónoma de Chihuahua, Chihuahua, México,

2016.

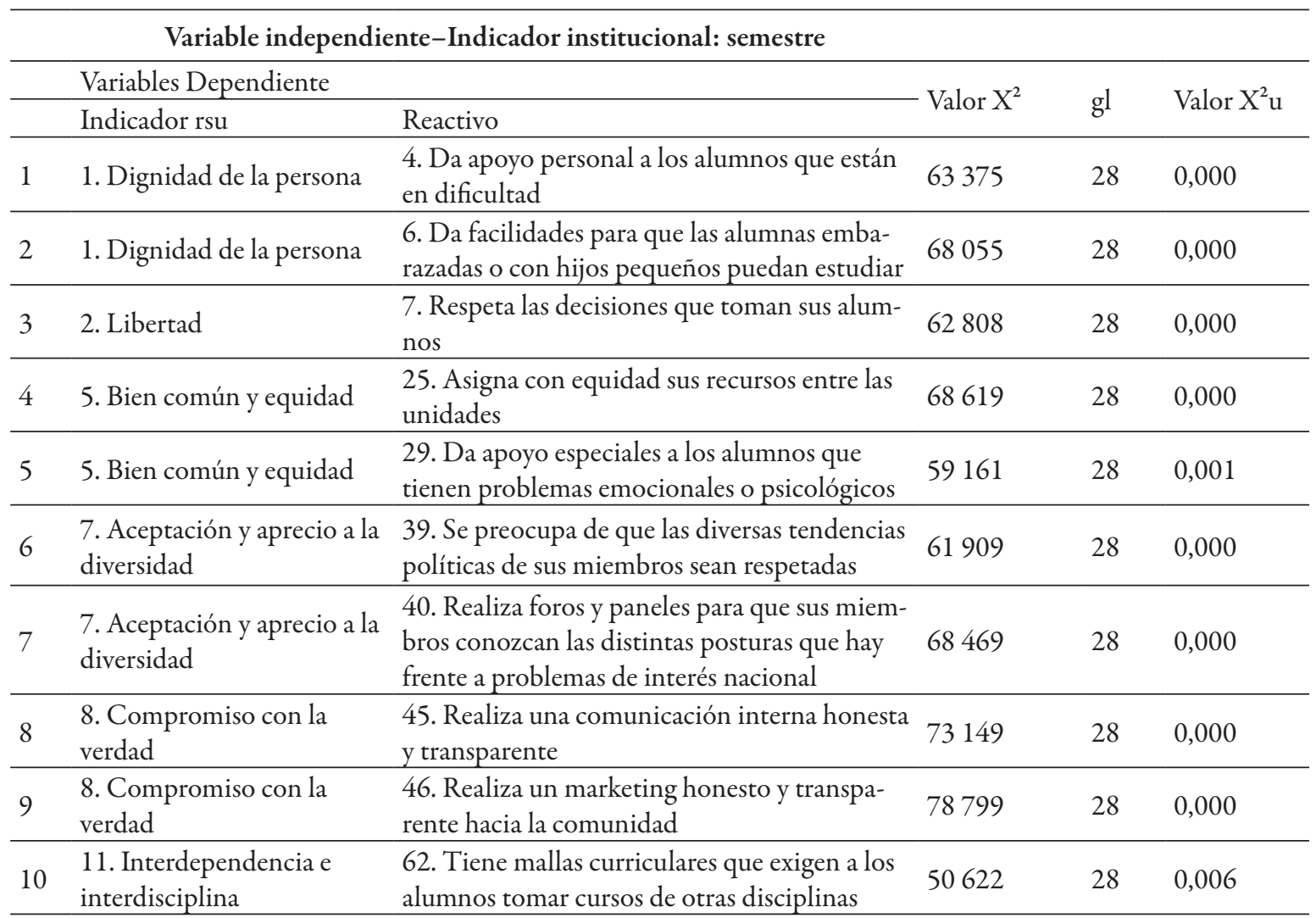

Fuente: Elaboración propia.

los reactivos. Destacan los indicadores 4 y 10 sin ninguna relación. Además, se observa que el Indicador 2 (Libertad) y el indicador 11 (Interdependencia e interdisciplina) mostraron relación con dos y tres reactivos respectivamente.

Los resultados de este cuadro muestran las 10 relacio- nes del indicador Semestre con 6 indicadores de la rsu y específicamente con cada uno de los reactivos. Destacan los indicadores 3, 4, 6, 9 y 10 sin ninguna relación. Además, se observa que los indicadores: 1 (Dignidad de la persona), 5 (Bien común y equidad), 7 (Aceptación y aprecio a la diversidad) y el indicador 8 (Compromiso con la verdad) Interdependencia e Interdisciplina) mostraron relación con dos reactivos respectivamente. 


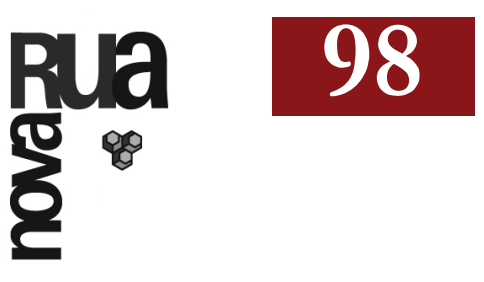

Cuadro 18. Resumen de resultados de las relaciones entre indicadores sociodemográficos e indicadores de la rsu. Estudio de RSU. Facultad de Enfermería y Nutriología de la Universidad Autónoma de Chihuahua, Chihuahua, México, 2016.

\begin{tabular}{|c|c|c|c|c|c|c|c|}
\hline \multirow{3}{*}{ Indicador RSU } & \multirow{3}{*}{$\%$} & \multirow{3}{*}{ Reactivos } & \multicolumn{5}{|c|}{ Indicadores sociodemográficos } \\
\hline & & & \multicolumn{2}{|c|}{$\begin{array}{l}\text { Indicadores } \\
\text { institucionales }\end{array}$} & \multicolumn{2}{|c|}{$\begin{array}{l}\text { Indicadores } \\
\text { personales }\end{array}$} & \multirow[b]{2}{*}{ Trab. } \\
\hline & & & Lic. & Sem. & Edad & Sexo & \\
\hline 1. Dignidad de la persona & 61 & 6 & 1 & 2 & & & \\
\hline 2. Libertad & 64 & 6 & 2 & 1 & & & \\
\hline 3. Ciudadanía, participación y democracia & 64 & 6 & 1 & & & & \\
\hline 4. Sociabilidad y solidaridad & 75 & 6 & & & & & \\
\hline 5. Bien común y equidad & 54 & 6 & 1 & 2 & & & \\
\hline 6. Medio ambiente y desarrollo sustentable & 70 & 6 & 1 & & & & \\
\hline 7. Aceptación y aprecio a la diversidad & 66 & 6 & 1 & 2 & & & \\
\hline 8. Compromiso con la Verdad & 62 & 6 & & 2 & & & \\
\hline 9. Integridad & 70 & 6 & 1 & & & & \\
\hline 10. Excelencia & 76 & 6 & & & & & \\
\hline \multirow[t]{2}{*}{ 11. Interdependencia e interdisciplina } & 72 & 6 & 3 & 1 & & & \\
\hline & 67 & 66 & 11 & 10 & 0 & 0 & 0 \\
\hline
\end{tabular}

Fuente: Elaboración propia

Se observa que los indicadores personales edad, sexo y trabajo no se relacionan con los indicadores de la rsu. Este resultado es importante ya que la percepción de las políticas de la uach, en el tema de rsu, no se ve afectada por ninguno de los indicadores personales.

Con relación a los indicadores institucionales, se puede apreciar, en el caso de Licenciaturas, que estas si mostraron relación con 11 de los 66 reactivos de la rsu. Estas relaciones se ubican en nueve de los 11 indicadores evaluados, es decir, no hubo relación con el indicador 4 y con el indicador 10 .

Para el caso del indicador Semestre, se detectaron 10 relaciones con los 66 reactivos de la rsu, distribuidos en seis de los 11 indicadores. Destacan los indicadores 3, 4, 6, 9 y 10 que no mostraron ningún tipo de relación.

Es importante señalar, en el caso de los indicadores Sociabilidad y solidaridad (4), y Excelencia (10), ambos no se ven afectados o relacionados por ningún indicador sociodemográfico y ambos mostraron va- lores superiores a $75 \%$ o más, en su grado de percepción.

Con relación a las licenciaturas (Nutriología y Enfermería), en el cuadro 19 se puede aprecia un patrón constante y uniforme sobre la percepción de la rsu en Nutriología, ya que esta presenta valores superiores de percepción en ocho de los 11 indicadores (en 11 reactivos) versus Enfermería.

Para el caso de los semestres (cuadro 20), los resultados muestran un patrón de percepción irregular con valores más o menos constantes en los primeros cuatro semestres. 
Cuadro 19. Diferencias de rsu por licenciatura en los indicadores con relación. Estudio de rsu. Facultad de Enfermería y Nutriología de la Universidad Autónoma de Chihuahua, Chihuahua, México, 2016.

\begin{tabular}{|c|c|c|c|c|}
\hline & Indicador rsu & $\begin{array}{c}\text { Enfermería Respuesta } \\
\text { positiva }(\%)\end{array}$ & \multicolumn{2}{|c|}{$\begin{array}{c}\text { Nutriología } \\
\text { Respuesta } \\
\text { positiva (\%) }\end{array}$} \\
\hline 1 & (1) Dignidad De La Persona & 61 & 65 & $\mathrm{~N}$ \\
\hline 2 & (2) Libertad & 68 & 77 & $\mathrm{~N}$ \\
\hline 3 & (2) Libertad & 55 & 63 & $\mathrm{~N}$ \\
\hline 4 & (3) Ciudadanía, participación y democracia & 38 & 46 & $\mathrm{~N}$ \\
\hline 5 & (4) Bien común y equidad & 59 & 48 & $\mathrm{E}$ \\
\hline 6 & (5) Medio ambiente y desarrollo sustentable & 74 & 68 & $\mathrm{E}$ \\
\hline 7 & (6) Aceptación y aprecio a la diversidad & 69 & 74 & $\mathrm{~N}$ \\
\hline 8 & (7) Integridad & 60 & 73 & $\mathrm{~N}$ \\
\hline 9 & (8) Interdependencia e interdisciplina & 80 & 90 & $\mathrm{~N}$ \\
\hline & (8) Interdependencia e interdisciplina & 67 & 72 & $\mathrm{~N}$ \\
\hline & (8) Interdependencia e interdisciplina & 69 & 75 & $\mathrm{~N}$ \\
\hline
\end{tabular}

Fuente: Elaboración propia

Cuadro 20. Percepción de la rsu por semestre (indicadores con relación). Estudio de rsu. Facultad de Enfermería y Nutriología de la Universidad Autónoma de Chihuahua, Chihuahua, México, 2016.

\begin{tabular}{ccccccccc}
\hline Semestre & $\mathbf{1}$ & $\mathbf{2}$ & $\mathbf{3}$ & $\mathbf{4}$ & $\mathbf{5}$ & $\mathbf{6}$ & $\mathbf{7}$ & $\mathbf{8}$ \\
\hline \% de percepción & 60.8 & 60.8 & 53.7 & 58.3 & 48.9 & 50.5 & 36.2 & 50.4 \\
\hline
\end{tabular}

Fuente: Elaboración propia.

A partir del quinto semestre, la precepción de la rsu disminuye y su comportamiento es más irregular.

\section{Conclusiones}

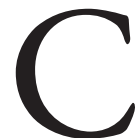
on base en las preguntas de investigación, los objetivos de estudio, hipótesis, resultados obtenidos y al contexto sobre el cual se llevó a cabo esta investigación, se concluye lo siguiente:
Pregunta 1. ¿Cómo es la percepción de la rsu por los estudiantes de la fen/uach?

Objetivo 1. Evaluar la percepción de la rsu por los alumnos de la fen/uach

Hipótesis 1. La percepción de la rsu por los alumnos de la fen/uach es positiva.

Resultados. El $40 \%$ de los estudiantes calificó la rsu con un 8 en la escala del 1 al 10 y $30 \%$ la evaluó con 9 (70 \%). La calificación promedio fue de 8 (cuadro 2). El $67 \%$ de la pobla- ción estuvo de acuerdo o muy de acuerdo en que se llevan a cabo las 66 acciones de los 11 indicadores rsu (cuadro 15).

Conclusión 1. La $\mathrm{H} 1$ se acepta de manera parcial asumiendo que el $40 \%$ de los estudiantes la apreció con un 8. Además, el $67 \%$ de los alumnos percibió que las prácticas de rsu son buenas; sin embargo, existe un $33 \%$ de ellos que no están de acuerdo con estas políticas. 
Pregunta 2. ¿Cuál es la relación entre los indicadores sociodemográficos de los estudiantes y los indicadores de la rsu?

Objetivo 2. Identificar la relación entre los indicadores sociodemográficos de los estudiantes de la fen con los indicadores de la rsu.

Hipótesis 2. No existe relación entre los indicadores sociodemográficos de los estudiantes de la FEN CON LOS INDICADORES DE LA RSU.

Resultados. Los indicadores personales edad, sexo y trabajo no mostraron ninguna relación con los 66 reactivos de los 11 indicadores de la RSU (CUADRo 18).

El indicador licenciatura se relacionó con 11 de los 66 reactivos de la RSU, donde los estudiantes de la carrera de $\mathrm{Nu}$ triología mostraron una mejor percepción, dominando nueve de los 11 indicadores de la rsu (cuadros 16 y 19).

En cuanto al semestre, este indicador mostró relación con 10 de los 66 reactivos de la rsu (cuadro 19). Sin embargo, no se detectó una tendencia clara o un comportamiento específico que indicará cierto dominio en la percepción de la rsu con relación al avance en la formación académica de los estudiantes (cuadros 17 y 20).

Conclusión 2. La H2 no se rechaza. Los indicadores sociodemográficos no tienen relación con los indicadores de la rsu. Los indicadores personales no mostraron ninguna relación. Aunque se identificaron ciertas relaciones de los indicadores institucionales (licenciatura y semestre) con los indicadores de la rsu, estas relaciones son mínimas y no se detectó una evidencia clara y contundente.

\section{RECOMENDACIONES}

pesar de que hay pro-
gramas y actividades
relacionadas con rs, es necesario generar acciones que vayan orientados a:

a) Incrementar la apreciación y mejorar el posicionamiento de la rsu en los estudiantes de la fen.

b) Reforzar los factores de éxito en el $67 \%$ de los estudiantes que han tenido percepción positiva sobre la rsu.

c) Desarrollar e implementar políticas de rsu que vayan orientados hacia el $33 \%$ de los estudiantes que no están de acuerdo y/o que no saben lo que se está haciendo con relación a la rsu.

En las futuras investigaciones es importante que se tomen en cuenta las políticas y acciones de la rs de la uach, las cuales deberán de estar orientadas hacia la estandarización de la percepción de la misma, es decir, se debe trabajar en los indicadores institucionales (licenciatura y semestre) de tal manera que no haya evidencias que manifiesten relaciones entre los indicadores socio demográfico y los indicadores de la rsu. Los estudiantes de la fen deben percibir de manera similar los que se está haciendo con la rsu.

Es importante la difusión de las actividades que hace constantemente la universidad en relación a desarrollar, constantemente la responsabilidad social entre los actores que deben de estar inmersos en ella (la responsabilidad) como son los estudiantes, los docentes y por supuesto, el personal administrativo, desde el rector, hasta el personal que se encuentra en cada una de las unidades académicas. 


\section{REFERENCIAS}

\section{BIBLIOGRÁFICAS}

Aponte, E. (2015). "La Res-

ponsabilidad Social de las

Universidades: Implica-

ciones para América Latina y el Caribe", Eduardo A. (ed.). Puerto Rico: Unesco-Iesalc.

Domínguez Pachón, M. J. (2009). Responsabilidad Social Universitaria.

Flont I., P. Gudiño, C. Salgado y A. Sánchez (2008). "Aproximaciones a las Responsabilidad Social Empresarial rse". Gestión y estrategia núm. 28, UAM, México.

Gaete, Q. R. (2006). “El proyecto Universidad construye país como referente de la rsu en Chile", rsu Chile Universidad construye país. Disponible en http:// www.rsuchile.cl/2013/09/ el-proyecto-universidadconstruye-pais.html

Gaete, Q. R. (2014). "La responsabilidad social universitaria como política pública: un estudio de caso". Documentos y aportes en Administración Pública y Gestión Estatal, vol. 14, núm. 22. Universidad Nacional del Litoral Santa Fe, Ar- gentina. ISSN: 1666-4124. Disponible en http:// www.redalyc.org/articulo. oa? $\mathrm{id}=337531584004$

Gasca-Pliego, E. y J. C. Olvera-García (2011). "Construir ciudadanía desde las universidades, Responsabilidad Social Universitaria y desafíos ante el siglo xxi". Convergencia. Revista de Ciencias Sociales, vol. 18, núm. 56. Universidad Autónoma del Estado de México. ISSN: 1405-1435. Disponible en http:// www.redalyc.org/articulo. oa?id=10516855002

Jiménez de la Jara, M. J. M. De Ferrari, C. Delpiano, y C. Ardiles (2004), "Proyecto UCP: Tres años construyendo país". Proyecto Universidad construye país. Disponible en http:// www.rsuchile.cl/

Serna G, M. (2016). "Llama Rector de la umich a que universidades induzcan al cambio", Observatorio Mexicano de Responsabilidad Social Universitaria, disponible en http:// omersu.org/1178-2/Universidad Construye País (2014).
Vallaeys, F. (2008), "rs en universidades". Pontifica Universidad Católica del Perú. Disponible en http:// www.iso26000peru.org/ accion.htm

Ysunza, M. y J. Molina (2010). "Principios éticos y Responsabilidad Social en la universidad". México: Universidad Autónoma Metropolitana. 